\title{
Unresectable Carcinoma Pancreas : Efficacy of Imaging in Diasnosing and Labeling them as 'Unresectable'
}

\author{
Md. Abdullah Al Farooq ${ }^{1 *}$ \\ M A Mushfiqur Rahman \\ Tania Tajreen ${ }^{2}$ \\ Eqramur Rahman ${ }^{3}$ \\ Md. Minhajuddin Sajid' \\ Mohammad $\mathrm{Ali}^{4}$
}

'Department of Pediatric Surgery Chittagong Medical College \& Hospital Chittagong, Bangladesh.

${ }^{2}$ Department of Medicine Chittagong Medical College \& Hospital Chittagong, Bangladesh.

${ }^{3}$ Consultant, Research and Development Ashford and St. Peter's Hospitals NHS Foundation Trust, Guildford Road, Chertsey Surrey, KT16 OPZ, London, UK.

${ }^{4}$ Department of Hepato-Biliary-Pancreatic Surgery BIRDEM Hospital, Shahbag

Dhaka, Bangladesh.

\begin{abstract}
Background: Carcinoma pancreas is being diagnosed increasingly with the help of conventional imaging like ultrasonography (USG), computerized tomography (CT) scan and magnetic resonance imaging (MRI).Imaging also gives the opportunity to assess resectability. In our country MRI and CT scan are not widely available and most of the pancreatic carcinoma is too advanced for curative surgical resection when diagnosed. These are unresectable carcinoma pancreas (UCP). Objectives: To evaluate the efficacy of imaging in diagnosing carcinoma pancreas and to assess resectability after comparing them with peroperative findings. Methods: This retrospective study was carried out in the department of Hepato-Biliary-Pancreatic Surgery in Bangladesh Institute for Research and Rehabilitation in Diabetic Endocrine and Metabolic disorders (BIRDEM) hospital, Dhaka, Bangladesh from July 2004 to June 2006 (2 years). After laparotomy findings and histopathological confirmation 50 patients were labeled as UCP. Among 50 patients male were 28 \& female patients were 22. Imaging modalities used before surgery was assessed and compared with per operative findings. USG were done in all patients and CTscan in 45 patients. MRI was done in 08 patients suspected clinically as pancreatic carcinoma where USG /CT scan had failed to reach a conclusion. Findings of the various imaging studies regarding diagnosis and unresectability were compared with per operative findings. Results: USG was able to diagnose $42(84 \%)$ pancreatic carcinoma patients with unresectibility in $29(69 \%)$. Forty five patients $(90 \%)$ were diagnosed by CT scan and could label $38(84.44 \%)$ as unresectable. MRI was $100 \%$ accurate to diagnose and label the entire 08 patient as unresectable carcinoma pancreas. Cumulative multimodal preoperative imaging was $91.33 \%$ accurate in diagnosing carcinoma pancreas and could tell the features of unresectibility in $73.59 \%$ patients. Conclusion: CT scan should be the primary imaging modality for diagnosing pancreatic carcinoma and its resectability. MRI is very promising for diagnosing and assessing UCP. Multimodal imaging is better than single imaging.
\end{abstract}

Key words: Pancreatic carcinoma; Unresectable; Imaging.

\section{INTRODUCTION}

Cancer pancreas is the $4^{\text {th }}$ leading cause of all cancer deaths. The peak incidence is in $5^{\text {th }}$ and $6^{\text {th }}$ decades of life ${ }^{1}$. In clinical practice, pancreatic cancer is synonymous with pancreatic ductal adenocarcinoma which constitute $90 \%$ of all the malignant tumors of the gland ${ }^{2}$. The incidence is $70 \%$ in the head, $30 \%$ in the body and tail of the pancreas $^{3}$. Accurate preoperative diagnosis \& the judgment of inoperability is still a dilemma ${ }^{4}$. Unfortunately at the time of presentation, $90-95 \%$ patients are unsuitable for curative resection. ${ }^{3}$ Pancreatic cancer is diagnosed on clinical presentation, laboratory investigations, tumor markers, imaging studies and some endoscopic procedures ${ }^{1}$. It is mandatory to have a cytological proof before the lesion is labeled as cancer pancreas ${ }^{4}$. Tremendous advancement has been achieved in the imaging of the deadly disease during the last two decades ${ }^{4}$. Imaging studies that are helpful in pancreatic cancer are ultrasonography (USG), endoscopic ultrasound (EUS), surgeon performed USG, computerized tomography (CT) scan, contrast CT scan, magnetic resonance imaging (MRI) and magnetic resonance cholangio pancreatography $(\mathrm{MRCP})^{1,4-6}$. USG is the first line test for evaluation and findings

\author{
Dr. Md. Abdullah Al Farooq \\ Assistant Professor \\ Department of Pediatric Surgery \\ Chittagong Medical College \& Hospital \\ Chittagong-4000, Bangladesh. \\ Mobile : +88-01815-002188 \\ Fax: +88-031628185 \\ E-mail: farooq71bd@yahoo.com
}


like hypoechoic mass, dilatation of both common bile duct and pancreatic duct (double duct sign) which suggest carcinoma pancreas. Overall sensitivity is $75 \%$ and specificity is also $75 \%$. Sensitivity and specificity for resectability is $63.5 \%$ and $83 \%$ respectively ${ }^{7}$. Most important CT scan findings in pancreatic carcinoma is ill defined hypodense pancreatic mass. A ssociate findings are dilatation of both common bile duct and pancreatic duct (double duct sign), fullness of the pancreatic head (loss of lobular appearance of pancreatic parenchyma) and atrophy of pancreatic tail ${ }^{7}$. MRI shows pancreatic carcinoma as hypointense area on $T_{1}$ weighted gadolinium enhanced fat suppressed sequences ${ }^{8}$.

Imaging may not detect small hepatic metastasis and peritoneal deposit which are readily detected by direct inspection of peritoneal cavity during laparotomy or laparoscopy ${ }^{9}$. Laparoscopic USG can image pancreatic tumor, local invasion, lymph nodes and distant metastasis to liver $^{9}$. A sequential approach consisting of CT scan as an initial test and EUS as a confirmatory technique seems to be the most reliable and cost minimizing strategy for pancreatic cancer imaging ${ }^{10}$. Findings contraindicating for curative resection are liver meatastases, celiac lymph node involvement, peritoneal implant, invasion of transverse colon and hepatic hilar lymph node involvement. Invasion of duodenum or distal stomach, peripancreatic lymph node and lymph node along porta hepatis that can be swept down along with the specimen does not contraindicate curative resection ${ }^{11}$. Even all test result shows resectability but practically only $78 \%$ of pancreatic cancers are resectable during laparotomy ${ }^{12}$. To the contrary, only five percent (5\%) pancreatic cancers are resectable that was labeled previously as unresectable $\mathrm{e}^{12}$.

In our country, we still depend on clinical findings, laboratory investigations \& USG as CT scan and MRI facilities are very limited. In this scenario, a considerable number of patients remain undiagnosed and untreated. Some patients are diagnosed at advanced stage of the disease. Present study was carried out in Bangladesh Institute for Research \& Rehabilitation in Diabetic Endocrine \& Metabolic disorders (BIRDEM) to evaluate current practice regarding imaging that have been used to diagnose pancreatic carcinoma and assess unresectablity.

\section{MATERIALS AND METHODS}

Hospital records of all pancreatic carcinoma patients admitted during the period of July 2004 to June 2006 (study period 2 years) in the department of Hepato-Biliary-Pancreatic Surgery in BIRDEM hospital, Dhaka, Bangladesh were retrospectively analyzed. Pre-operatively labeled unresectable carcinoma pancreas patients that underwent laparotomy with histopathological proof during this period were included for the study. Total fifty patients were labeled as 'unresectable carcinoma pancreas' $(n=50)$; male $28\left(n_{1}=28\right)$ and female 22 $\left(n_{2}=22\right)$. All patients were evaluated by USG and CT scan. M RI was done where USG /CT scan had failed to give any clue about the diagnosis in clinically suspected 8 carcinoma pancreas patients. USG findings suggested to diagnose carcinoma pancreas was hypoechoic mass in pancreas and dilatation of both common bile duct and pancreatic duct. In CT scan ill defined hypodense pancreatic mass and dilatation of both common bile duct and pancreatic duct was accepted as carcinoma pancreas. M RI showing hypointense area in pancreas on $\mathrm{T}_{1}$ weighted image sequences was considered as carcinoma pancreas. Imaging findings accepted to label pancreatic carcinoma 'unresectable' were liver meatastasis, the mesenteric involvement, mesenteric vascular invasion, portal venous invasion, celiac lymph node involvement, peritoneal implant, ascites, gross invasion to transverse colon, stomach, duodenum, behind the pancreas and hepatic hilar lymph node involvement. These findings were compared with per operative findings and summarized. Chi-square $\left(\alpha^{2}\right)$ test was applied to show the significance in difference between imaging findings and per operative findings, $p$-value $<0.01$ was taken as significant.

\section{RESULTS}

Age distribution: Patients most commonly presented between the ages of $56-60$ years (16 patients, $32 \%$ ). Only $2(4 \%)$ patients presented between $45-50$ years of age. The median age of presentation is between 51 and 70 years (80\%).

Table-1 shows features of preoperative imaging studies and peroperative findings. It was noted that $47(94 \%)$ patients had multiple features of unresectability and only $3(6 \%)$ had single feature in favour of unresectable tumour.

Table 1 : Imaging and per operative findings of UCP

\begin{tabular}{lcccc} 
& \multicolumn{4}{c}{ Findings } \\
\cline { 2 - 5 } & USG & CT & MRI & Per-operative \\
$\begin{array}{l}\text { Total number } \\
\text { of patient }\end{array}$ & $50(100 \%)$ & $50(100 \%)$ & $8(16 \%)$ & $50(100 \%)$ \\
assessed & & & & \\
$\begin{array}{l}\text { Diagnosed as } \\
\text { pancreatic } \\
\text { cancer with } \\
\text { percentage }\end{array}$ & $42(84 \%)$ & $45(90 \%)$ & $8(100 \%)$ & $50(100 \%)$ \\
& & & & \\
\end{tabular}

\begin{tabular}{|c|c|c|c|c|}
\hline $\begin{array}{l}\text { Location of } \\
\text { the lesion }\end{array}$ & (Out of 42) & (Out of 45) & (Out of 8) & (Out of 50) \\
\hline Head & $33(78.57 \%)$ & $33(73.33 \%)$ & $6(75 \%)$ & $38(76 \%)$ \\
\hline Body & $08(19.04 \%)$ & $10(22.22 \%)$ & $2(25 \%)$ & $09(18 \%)$ \\
\hline Tail & $01(2.38 \%)$ & $02(04.44 \%)$ & - & $03(6 \%)$ \\
\hline Ascites & $8(19.07 \%)$ & $10(22.22 \%)$ & $1(16.67)$ & $12(24 \%)$ \\
\hline $\begin{array}{l}\text { Local } \\
\text { extension } \\
\text { of Tumor }\end{array}$ & $7(16.66 \%)$ & $11(24.44 \%)$ & $2(25 \%)$ & $16(32 \%)$ \\
\hline $\begin{array}{l}\text { B ehind the } \\
\text { pancreas }\end{array}$ & $4(9.52 \%)$ & $6(13.33 \%)$ & $2(25 \%)$ & $8(16 \%)$ \\
\hline Stomach & $1(2.38 \%)$ & $3(6.66 \%)$ & - & $4(8 \%)$ \\
\hline Duodenum & $2(4.76 \%)$ & $1(2.22 \%)$ & $1(12.5 \%)$ & $3(6 \%)$ \\
\hline Transverse colon & - & $1(2.22 \%)$ & - & $1(2 \%)$ \\
\hline
\end{tabular}




\begin{tabular}{|c|c|c|c|c|}
\hline Involvement & $13(30.95 \%)$ & 20 (44.44\%) & $5(62.5 \%)$ & $46(92 \%)$ \\
\hline Liver & $5(11.90 \%)$ & $6(13.33 \%)$ & $1(12.5 \%)$ & $7(14 \%)$ \\
\hline M esenteric vessel & $1(2.38 \%)$ & $2(4.44 \%)$ & $1(12.5 \%)$ & $6(12 \%)$ \\
\hline Portal vein & $2(4.76 \%)$ & $1(2.22 \%)$ & $1(12.5 \%)$ & $4(08 \%)$ \\
\hline $\begin{array}{l}\text { Hepatic hilar } \\
\text { lymph node }\end{array}$ & $4(9.52 \%)$ & $7(16.66 \%)$ & $2(25 \%)$ & $10(20 \%)$ \\
\hline Celiac node & $1(2.38 \%)$ & $1(2.22 \%)$ & - & $4(8 \%)$ \\
\hline The mesentery & - & $2(4.44 \%)$ & - & $6(12 \%)$ \\
\hline Peritoneum & - & $1(2.22 \%)$ & - & $9(18 \%)$ \\
\hline Fixed lesion & - & - & - & $41(82 \%)$ \\
\hline M obile lesion & - & - & - & $9(18 \%)$ \\
\hline $\begin{array}{l}\text { Features of } \\
\text { unresectibility } \\
\text { found with } \\
\text { their percentage }\end{array}$ & $29(69 \%)$ & $38(84.44 \%)$ & $08(100 \%)$ & $50(100 \%)$ \\
\hline
\end{tabular}

USG was used in all suspected cases $(n=50)$. USG was able to diagnose 42 patients (84\%) with pancreatic carcinoma. Out of 42 , USG could delineate features of unresectibility in 29 patients $(69 \%)$. It had missed $8(16 \%)$ patients with pancreatic cancer \& could not say about unresectibility in $13(31 \%)$ patients.

CT scan was done in all patients and it diagnosed 45 patients (90\%) with pancreatic carcinoma. Out of 45 , CT scan could delineate the features of unresectibility in 38 patients $(84.44 \%)$. It had missed $5(10 \%)$ patients with pancreatic carcinoma and unable to say about unresectibility in 07 (15.55\%) patients.

MRI was carried out only in 08 patients. MRI was $100 \%$ accurate to diagnose the patients as carcinoma pancreas and had found features of unresectibility in all (100\%).

Per operative findings: Most of the lesions were found at the head of the pancreas (38 patients (76\%). Body (09 patients, $18 \%$ ) and tail (03 patients, 6\%) was also involved. In 41 patients $(82 \%)$ lesions were fixed and in 09 patients $(17.5 \%)$ were mobile. Local extension was noted in $16(32 \%)$ patients. Regional lymph node involvement was noted in 14 (28\%) patients. Invasion of the mesentery $(6,12 \%)$, mesenteric vessels $(6,12 \%)$, portal vein $(4,8 \%)$ and liver $(7,14 \%)$ were also noted. Ascites (12, 24\%) and peritoneal seedling (09, 18\%) were also evident.

Considering all the imaging, cumulative multimodal preoperative imaging was accurate in diagnosing carcinoma pancreas in $91.33 \%$ and could tell the features of unresectibility in $73.59 \%$ patients.

\section{DISCUSSION}

The incidence of pancreatic cancer has tripled over the last 40 years ${ }^{2}$. The incidence of pancreatic cancer is 10 per 100,000 per year with equal involvement of both sexes and unfortunately, at the time of presentation $90-95 \%$ patients are unsuitable for curative resection ${ }^{3}$. In the recent years more women are being diagnosed with this cancer in increasing frequency ${ }^{2}$. In our study, male (28 patients) are affected more than the female (22 patients) and ratio is 1.27:1. Yeo and Cameron also noted male sex is more vulnerable to pancreatic cancer ${ }^{4}$.

Most of the pancreatic cancer patients are aged ${ }^{3}$ and the peak incidence is at $5^{\text {th }}$ and $6^{\text {th }}$ decade 1 . In our study, 40 patients $(80 \%)$ were aged $51-70$ years and no patient was diagnosed before 45 years of age.

On laparotomy, frequency of involvement of the region of the pancreas was, head (38 patients, $76 \%$ ), body (09 patients, 18\%), tail (03 patients, 6\%) which is near to the findings of Russel ${ }^{3}$ (head $70 \%$, body and tail $30 \%$ ).

USG is $83 \%$ sensitive and $99 \%$ specific in diagnosing advanced pancreatic cancer ${ }^{13}$. USG is the first line diagnostic tool in patient having pancreatic tumor. It is $90 \%$ sensitive in diagnosing pancreatic cancer ${ }^{14}$. In our study, USG was able to diagnose 42 patients (84\%) with pancreatic cancer and missed 8 $(16 \%)$ patients which was confirmed in laparotomy. Regarding diagnosis, USG is moderately accurate ( $p$ value $<0.01$ ). USG was able to reveal ascites in $8(19.07 \%)$ patients, local extension in $7(16.66 \%)$, involvement of liver in $5(11.9 \%)$, mesenteric vessel in $1(2.38 \%)$, portal vein invasion in $2(4.76 \%)$, hepatic hilar lymph node in $4(9.52 \%)$ and celiac node in $1(2.38 \%)$. In comparison, laparotomy revealed ascites in $12(24 \%)$ patients, local extension in 16(32\%), involvement of liver in $7(14 \%)$, mesenteric vessel in $6(12 \%)$, portal vein invasion in $4(8 \%)$, hepatic hilar lymph node in $10(20 \%)$ and celiac node in $4(8 \%)$, the mesentery in $6(12 \%)$ and peritoneal involvement in 9 $(18 \%)$. From these data, it is clear that USG has failed to detect local extension and involvement of liver, mesenteric vessels, portal vein, regional lymph nodes in significant percentage of patients ( $p$ value $<0.01$ ). To label pancreatic carcinoma as unresectable, the efficacy of USG was found poor preoperatively as $29(69 \%)$ patients were labeled UCP which was significantly lower than actual UCP (50 patients, $100 \%$ ). But high frequency USG can outline the pancreas easily and it is safe, easily available, non-invasive and brief; it can also detect dilated bile duct, hepatic metastasis, ascites or coexistent gall stone ${ }^{5}$. Surgeon performed USG provide rapid and accurate diagnosis of hepatobiliary pathology and may constitute to the management of hepatobiliary disease ${ }^{6}$.

CT scan diagnosed 45 patients $(90 \%)$ of pancreatic carcinoma and had missed $5(10 \%)$. A s a diagnostic tool, CT scan is nearly accurate ( $p$ value $<0.01)$. CT was able to reveal $10(22.22 \%)$ patients with ascites which is near to laparotomy findings (12 patients, 24\%). It was able to comment about the local extension in 11 (24.44\%), which is significantly lower than laparotomy findings (16 patients, 32\%). CT scan noted 20 patients $(44.44 \%)$ with organ involvement which is also significantly lower than actual $(46,92 \%)$. CT scan labeled 38 $(84.44 \%)$ patients as UCP in contrast to $100 \%$ patient with UCP. CT scan has highest accuracy in assessing primary tumor $(75 \%)$, loco regional extension (74\%), vascular invasion (88\%) and distant metastasis $(88 \%)^{10}$. CT scan shows pancreatic lesion in $95 \%$ cases. Contrast medium can be used before the study to distinguish tumor from normal tissue ${ }^{5}$. CT can detect $90 \%$ UCP ${ }^{12}$. Present study showed better delineation of pancreatic carcinoma than that of Soriano et al ${ }^{10}$. probably due to delay in referral which made it easily detectable? In this study, efficacy of CT scan (90\%) in diagnosing UCP is lower than shown by Doherty and way $(95 \%)^{1}$ and Warshaw $(90 \%)^{12}$. 
MRI diagnosed all the 8 patients (100\%) as pancreatic carcinoma and had not missed any patient. M RI had revealed 1 (16.67\%) patient with ascites but actually $12(24 \%)$ patients had ascites. It was able to comment about the local extension in $02(25 \%)$ and had noted 5 patients $(62.5 \%)$ with organ involvement. Out of 8 pancreatic carcinoma patients detected, M RI was able to delineate the features of unresectibility in all $(100 \%)$ of them. M RI findings are same as laparotomy findings regarding diagnosis or unresectability. MRI clearly displays pancreas and its duct system ${ }^{4}$. MRCP can replace ERCP in diagnostic purpose 4 . M RI is highly accurate detecting vascular invasion in $96 \%$ patient $^{10}$.

In the present study diagnostic yield of CT scan for UCP is higher than USG. To assess unresectability, CT scan is significantly superior to USG. Although MRI seems to be a better imaging modality than USG or CT scan in diagnosing pancreatic carcinoma and to label them as UCP, it is difficult to make an inference from this as small number of patients were found to be evaluated by MRI. But Warshaw found no difference or any added benefit from CT scan in detecting UCP. ${ }^{12}$ M RCP, Laparoscopy or laparoscopic US were not used in any patient either for diagnosis or assessment of unresectability. Considering all the imaging, cumulative multimodal imaging was $91.33 \%$ accurate in diagnosing carcinoma pancreas and can tell the features of unresectibility in $73.59 \%$ patients.

\section{CONCLUSION}

Evaluation of pancreatic carcinoma and its unresectability should be done by CT scan. USG is moderately good alternative only to diagnose pancreatic carcinoma but poor in assessing unresectability. MRI is a promising diagnostic tool both for diagnosis and assessment of unresectibilty but not easily available. Multimodal imaging is better than single imaging modality.

\section{REFERENCES}

1. Doherty GM, Way LW. Pancreas. In:Way LW, D oherty GM , editors. Current surgical diagnosis and treatment.N ew York: L ange Medical books; 2003.pp.602-12.

2. M oosa AR, M ouvet M, Gmagami RA. Disorder of pancreas. In: Cuschieri SA, Stelle RJC, M oosa AR, editors. Essential surgical practice. London: A rnold; 2002. pp. 477-576.

3. Russel RCG. The pancreas. In: Russl RCG, William N, Bulstrode CJK, editors. Short practice of surgery. L ondon: A rnold; 2004. pp. 1114-32.

4. Yeo CJ, Cameron JL. Exocrine pancreas. In: Townsend CM, B eauschamp RD, Evers B M, M ottok KL, editors. Sabiston textbook of surgery, the biological basis of modern surgical practice. Philadelphia: Saunders; 2001. pp.1112- 41.

5. M ayo clinic (US). Pancreatic cancer. N ew York : The institute; 2007.

6. Kell MR, A herne NJ, Coffey C, Power CP, K irwan WO, Redmond HP. Emergency surgeons' performed hepatobiliary ultrasonography. B ritish Surg J. 2002; 89(11): 1402-4.

7. Delden O V, Smithuis R. Pancreas-carcinoma. The radiology assistant 2006. www.radiologyassistant.nl/en/.../pancreascarcinoma.html

8. Miller F H, Rini N J, Keppke K L. M RI of adenocarcnoma of the pancreas. AJR 2006;187: 365-74.

9. Garden 0 J. Pancreatic cancer: Laparoscopic staging. In: Carter D, Russel RCG, Pitt H A, Bismuth H, editors. Rob and Smith's operative surgery, hepatobiliary and pancreatic surgery. L ondon, N ew York: Chapman and Hall M edical; 1996. pp. 576-84.

10. Soriano A, Castells A, Ayuso C, de Caralt M T, Gines M A, Real MI, et al. Preoperative staging and tumour resectability, assessment of pancreatic cancer: prospective study comparing EUS, helical computed tomography, magnetic resonance imaging and angiography. A m J Gastroentero. 2004; 99(3): 492-501.

11. Fisher W E, A nderson D K, B ell R H, Saluja AK, B runicardi FC. Panceas. In: Brunicardi F C, Anderson D K, Billiar T K et al., editors. Schwartz's principles of surgery. New York: M CGraw-Hill M edical Publishing division; 2005. pp. 1221-96.

12. Warshaw AL, Gu ZY, Witterberg J, Waltman AC. Pre-operative staging and assessment of resectability of pancreatic cancer. A rch Surg 1990; 125(2): 230-3.

13. Maringhini A, Ciambra M, Raimondo M, B acelliere P, Grasso R, Dardanoni G. Clinical presentation and Ultrasonography in the diagnosis of pancreatic cancer. Pancreas 1993; 8(2): 146-50.

14. K arson B-M , Ekbom A, L indgren PG, Kallskog V, Rastad J. A bdominal us for diagnosis of pancreatic tumour: prospective cohort analysis. Radiology1999; 213:107-11. 